\title{
Mathematical model of dynamics of two-coil synchronous electromagnetic impact machine with spring reversal of striker
}

\author{
V.Yu. Neyman \\ Novosibirsk State Technical University \\ Novosibirsk, Russian Federation \\ nv.nstu@ngs.ru
}

\author{
L.A. Neyman \\ Novosibirsk State Technical University \\ Novosibirsk, Russian Federation \\ neyman31@gmal.com
}

\author{
A.V. Markov \\ Novosibirsk State Technical University \\ Novosibirsk, Russian Federation \\ alexeimarkov@list.ru
}

\begin{abstract}
The development of modern methods of analysis and synthesis of electromechanical systems, including the main structural link of which is an electromagnetic drive, causes an increased interest in the creation and improvement of mathematical models of machines and devices that most objectively reflect their dynamic state in various technological processes $[1,2]$. The increase of the efficiency of such electric drives is possible only if the interconnected parameters of the electrical, magnetic and mechanical subsystems are rationally selected, and their operation is analyzed, and the need for dynamic calculation arises at the engineering stage. An object of research is an electromagnetic shock unit of a two-coil synchronous electromagnetic machine with a spring reversal of a striker containing a multimass oscillatory system with elastic connections and receiving power from a single-phase voltage source of industrial frequency by a half-wave rectification scheme. A mathematical model of the dynamics of an electromagnetic shock unit has been created, which provides broad possibilities for a comprehensive analysis of electromechanical processes during the excitation of periodic shock impulses of forces and interaction with a deformed medium. The advantage of the model is the ability to take into account a large number of interrelated factors, depending on the speed of the striker and the frequency of the shock impulses of forces, the degree of mobility of the inertial masses and the properties of the elastic bonds of the mechanical system, the magnetic properties of materials, accompanied by various kinds of energy losses. The methods and means of structural modeling in Matlab Simulink are an example of a numerical implementation of a shock-junction model with energy losses. The verification of the mathematical model is performed by comparing the calculated values of the output indicators with the results obtained on the physical model.
\end{abstract}

Keywords- impact knot, synchronous electromagnetic machine, mathematical model, mechanical oscillation system, elastic bonds, Lagrange equations of the second kind.

\section{INTRODUCTION}

The development of modern methods of analysis and synthesis of electromechanical systems, including the main structural link of which is the electromagnetic drive, implies an increased interest in the creation and improvement of the mathematical models of machines and devices that most objectively reflect their dynamic state in various technological processes [1,2]. A promising sphere of research in this area is the improvement of machines and mechanisms with the increased impact energy based on the electromagnetic drive of oscillatory motion, operating in resonance and near-resonance regimes with a frequency of $100 \mathrm{~Hz}$ [1-4]. The increase in the efficiency of such electric actuators is possible only on condition of a rational choice of the interconnected parameters of the electric, magnetic and mechanical subsystems, for the analysis of their work, even at the design stage, one needs dynamic calculation [3-5].

Current recommendations and techniques for the design of such systems are based in the majority on static approaches, which limit the possibilities of analyzing the working processes of electromechanical oscillatory systems, operating in transient and quasi-steady-state regimes [1,2].

The aim of the work is to develop a mathematical model of the dynamic state of a two-coil synchronous electromagnetic impact machine with a spring-loaded reverser of the striker, which provides opportunities for conducting a comprehensive analysis of interrelated electromechanical processes in transient and quasi-steady operating regimes.

\section{ELECTROMAGNETIC SHOCK UNIT WITH ELASTIC BEARING UNBEATABLE REVERSE}

An object of research is the design of an electromagnetic shock unit of a two-coil synchronous electromagnetic impact machine (Fig. 1) containing independent coils of forward 1 and reverse 2 located inside the magnetic circuit 3 and providing alternate acceleration of the striking mass of the striker 4 by electromagnetic forces in the forward and backward direction. Striker 4, reciprocating, alternately interacts with buffer spring 5 , which stops and reverses it, and working tool 6 which interacts with deformable medium 7 . Steady coupling of the electromagnetic shock unit to the 
working tool and to the deformable medium is provided by the pressing force.

The implementation of the working cycle is carried out with a constant effect of the electromagnetic forces of the coils receiving power from a single-phase voltage source in a halfwave rectification scheme.

The full operating cycle of the electromagnetic shock unit is carried out during one period of the voltage of the supply source, which provides a synchronous frequency of strokes striker at a frequency of $f=50 \mathrm{~Hz}$ $n_{\mathrm{imp}}=\frac{60 f}{2 p}=3000 \mathrm{imp} / \mathrm{min}$ and the duration of the working cycle of $t_{\mathrm{c}}=\frac{2 p}{f}=0,02 \mathrm{~s}$, where $2 p=1-$ number of voltage periods during the working cycle.

At the initial stage of the calculation, the process of motion of a mechanical system is considered as the result of superimposing forced and free vibrations excited by an external periodic force.

The mechanical and magnetic subsystems are connected by the functional dependence of electromagnetic force $f_{\mathrm{em} 1}=f\left(i_{1}, x_{1}\right)$ and $f_{\mathrm{em} 2}=f\left(i_{2}, x_{1}\right)$ of the current flowing through winding $i_{1}, i_{2}$ and position of striker $x_{1}$, and the electric and magnetic subsystems - by the functional dependence of the magnitude of flux linkage $\psi_{1}=f\left(i_{1}, x_{1}\right)$ and $\psi_{2}=f\left(i_{2}, x_{1}\right)$.

The connection between the electric and magnetic subsystems is described by the equation of electric equilibrium:

$$
\begin{aligned}
& u_{1}(t)=i_{1} r_{1}+\frac{d \psi_{1}\left(i_{1}, x_{1}\right)}{d t}, \\
& u_{2}(t)=i_{2} r_{2}+\frac{d \psi_{2}\left(i_{2}, x_{1}\right)}{d t},
\end{aligned}
$$

where $u_{1}(t), u_{2}(t)$ - voltage on the winding of the first and second coils; $r_{1}, r_{2}$ - active resistance of the winding of the first and second coils.

In accordance with the established relations in the construction of the shock unit (Fig. 1) and the general forces, acting in the system in Fig. 2, the calculated dynamic scheme of the mechanical oscillatory system is shown.

The linear displacement of the center of mass of the striker $x_{1}$ mass $m_{1}$, the working tool $x_{2}$ with mass $m_{2}$, the electric drive $x_{3}$ with mass $m_{3}$ are considered as generalized coordinates that determine the position of the mechanical system in space

Assuming the position of stable equilibrium as the reference point of the generalized coordinate and for the zero level of the potential energy, the authors consider small oscillations of the dynamical system with respect to the position of its static equilibrium.

The oscillations of the system in space will describe the corresponding to the $i$-th generalized coordinates $x_{1}, x_{2}$ and $x_{3}$ from the time accounted from the equilibrium position.

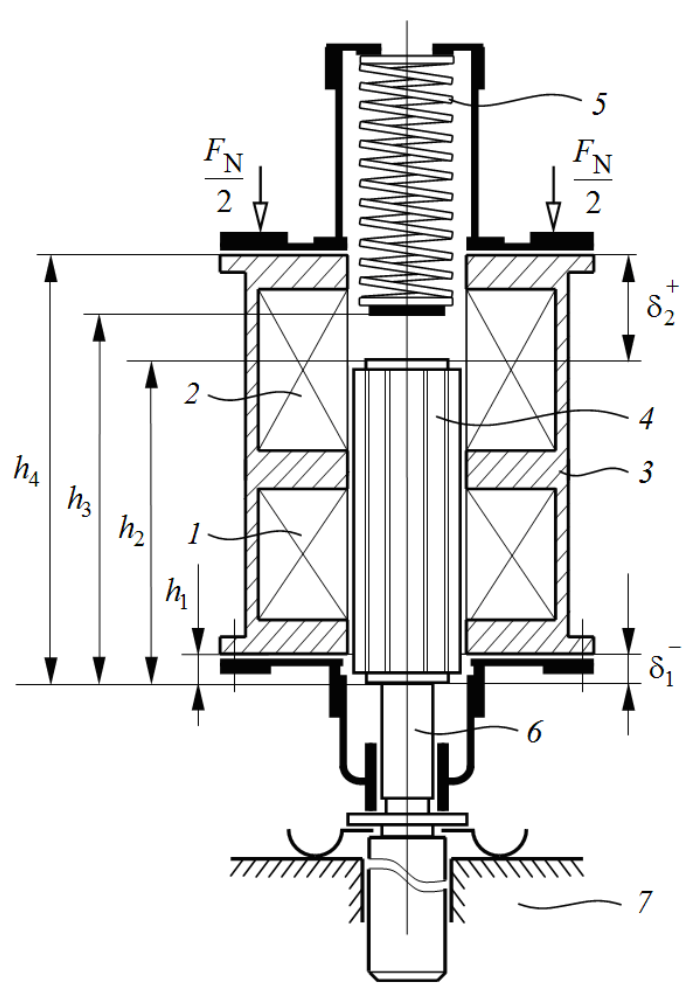

Fig. 1. Electromagnetic shock node

The equations of motion of a mechanical system are obtained based on Lagrange equations of the second kind:

$$
\frac{d}{d t}\left(\frac{\partial T}{\partial \dot{x}_{i}}\right)-\frac{\partial T}{\partial x_{i}}=-\frac{\partial \Pi}{\partial x_{i}}-\frac{\partial \Phi}{\partial \dot{x}_{i}}+Q_{i}, \quad i=1,2, . ., n
$$

where $T$ - kinetic energy of the system; $\Pi$ - potential energy of the system; $\Phi$ - dissipative function of the system (the Rayleigh function); $Q_{i}$ - generalized force of external influences of the system, corresponding to the $i$-th generalized coordinate; $x_{i}$ - generalized coordinate; $\dot{x}_{i}$ generalized speeds; $n$ - number of degrees of freedom of the mechanical system $(n=3)$.

Considering the motion of the masse $m_{1}, m_{2}$ and $m_{3}$ with respect to the chosen generalized coordinates, the authors successively define the quantities entering the Lagrange equation: 

masses

- kinetic energy of a mechanical system for progressive

$$
T=\frac{m_{1} \dot{x}_{1}^{2}}{2}+\frac{m_{2} \dot{x}_{2}^{2}}{2}+\frac{m_{3} \dot{x}_{3}^{2}}{2}
$$

- potential energy of elastic bonds of a mechanical system:

$$
\Pi=\frac{k_{1}\left(x_{1}-x_{3}\right)^{2}}{2}+\frac{k_{2} x_{2}^{2}}{2}+\frac{k_{3}\left(x_{3}-x_{2}\right)^{2}}{2},
$$

where $k_{1}, k_{2}, k_{3}$ - stiffness coefficients of elastic bonds;

- $\quad$ dissipative function (Rayleigh function):

$$
\Phi=\frac{b_{1}\left(\dot{x}_{1}-\dot{x}_{3}\right)^{2}}{2}+\frac{b_{2} \dot{x}_{2}^{2}}{2}+\frac{b_{3}\left(\dot{x}_{3}-\dot{x}_{2}\right)^{2}}{2},
$$

where $b_{1}, b_{2}, b_{3}$ - coefficients of viscous friction of elastic bonds;

- generalized forces of interactions of the system, corresponding to generalized coordinates:

$$
\begin{aligned}
& Q_{x_{1}}=-f_{\mathrm{em} 1}\left(i_{1}, x_{1}\right)+f_{\mathrm{em} 2}\left(i_{2}, x_{1}\right)-f_{\mathrm{fr}_{13}} \operatorname{sign} \dot{x}_{1} ; \\
& Q_{x_{2}}=-f_{\mathrm{fr}_{23}} \operatorname{sign} \dot{x}_{2} \\
& Q_{x_{3}}=f_{\mathrm{em} 1}\left(i_{1}, x_{1}\right)-f_{\mathrm{em} 2}\left(i_{2}, x_{1}\right)+ \\
& +\left(f_{\mathrm{fr}_{13}}-f_{\mathrm{fr}_{12}}\right) \operatorname{sign} \dot{x}_{3}-F_{\mathrm{N}},
\end{aligned}
$$

where $f_{\mathrm{em} 1}\left(i_{1}, x_{1}\right), f_{\mathrm{em} 2}\left(i_{2}, x_{1}\right)$ - forcing the electromagnetic force of the coils; $f_{\mathrm{fr}_{12}}, f_{\mathrm{fr}_{13}}, f_{\mathrm{fr}_{23}}$ - dry friction forces; $F_{N}$ constant value of pressing force;

$\stackrel{\bullet}{\text { rivatives: }}$

partial derivatives of kinetic energy and time

$$
\begin{array}{ll}
\frac{d}{d t}\left(\frac{\partial T}{\partial \dot{x}_{1}}\right)=m_{1} \ddot{x}_{1}, & \frac{\partial T}{\partial x_{1}}=0 ; \\
\frac{d}{d t}\left(\frac{\partial T}{\partial \dot{x}_{2}}\right)=m_{2} \ddot{x}_{2}, & \frac{\partial T}{\partial x_{2}}=0 ; \\
\frac{d}{d t}\left(\frac{\partial T}{\partial \dot{x}_{3}}\right)=m_{3} \ddot{x}_{3}, & \frac{\partial T}{\partial x_{3}}=0 ;
\end{array}
$$

\section{generalized coordinates:}

partial derivatives of potential energy from

$$
\frac{\partial \Pi}{\partial x_{1}}=k_{1}\left(x_{1}-x_{3}\right)
$$

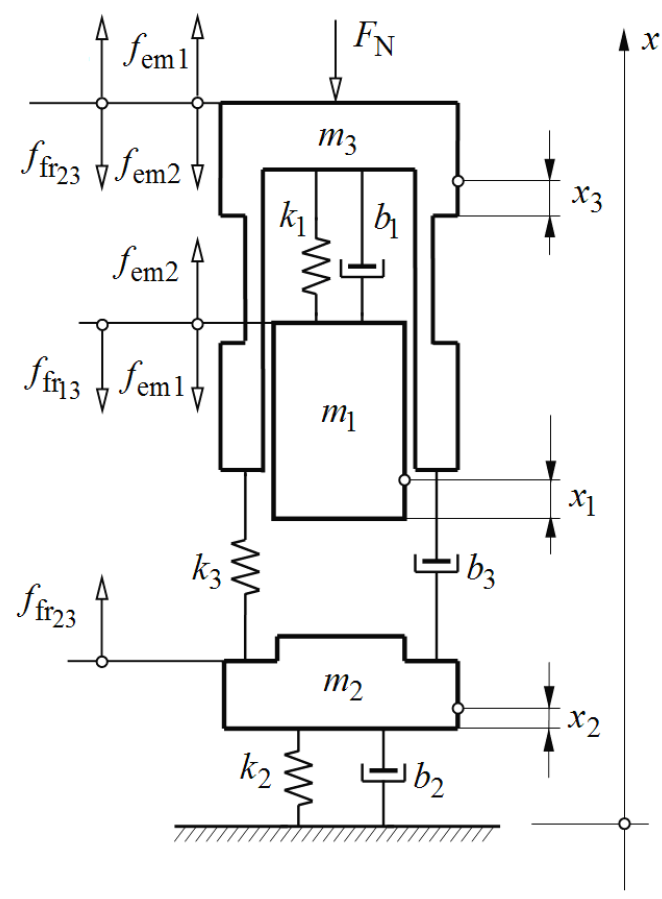

Fig. 2. The calculated dynamic scheme of the mechanical system

$$
\begin{aligned}
& \frac{\partial \Pi}{\partial x_{2}}=k_{2} x_{2}-k_{3}\left(x_{3}-x_{2}\right) ; \\
& \frac{\partial \Pi}{\partial x_{3}}=-k_{1}\left(x_{1}-x_{3}\right)+k_{3}\left(x_{3}-x_{2}\right) ;
\end{aligned}
$$

- partial derivatives of the dissipative function by the generalized velocity:

$$
\begin{aligned}
& \frac{\partial \Phi}{\partial \dot{x}_{1}}=b_{1}\left(\dot{x}_{1}-\dot{x}_{3}\right) ; \\
& \frac{\partial \Phi}{\partial \dot{x}_{2}}=b_{2} \dot{x}_{2}-b_{3}\left(\dot{x}_{1}-\dot{x}_{2}\right) ; \\
& \frac{\partial \Phi}{\partial \dot{x}_{3}}=-b_{1}\left(\dot{x}_{1}-\dot{x}_{3}\right)+b_{3}\left(\dot{x}_{3}-\dot{x}_{2}\right) .
\end{aligned}
$$

Introducing the derived values of the derivatives of the kinetic energy and the generalized forces, corresponding to 
the potential energy, the scattering energies and external influences, into Lagrange's equations, the differential equations of motion of the mechanical system of the model were deduced:

$$
\left\{\begin{aligned}
m_{1} \ddot{x}_{1}= & -k_{1}\left(x_{1}-x_{3}\right)-b_{1}\left(\dot{x}_{1}-\dot{x}_{3}\right)-f_{\mathrm{em} 1}\left(i_{1}, x_{1}\right)+ \\
& +f_{\mathrm{em} 2}\left(i_{2}, x_{1}\right)-f_{\mathrm{fr}_{13}} \operatorname{sign} \dot{x}_{1} \\
m_{2} \ddot{x}_{2}= & -k_{2} x_{2}+k_{3}\left(x_{3}-x_{2}\right)-b_{2} \dot{x}_{2}+b_{3}\left(\dot{x}_{1}-\dot{x}_{2}\right)+ \\
& +f_{\mathrm{fr}_{23}} \operatorname{sign} \dot{x}_{2} ; \\
m_{3} \ddot{x}_{3}= & k_{1}\left(x_{1}-x_{3}\right)-k_{3}\left(x_{3}-x_{2}\right)+b_{1}\left(\dot{x}_{1}-\dot{x}_{3}\right)- \\
& -b_{3}\left(\dot{x}_{3}-\dot{x}_{2}\right)+f_{\mathrm{em} 1}\left(i_{1}, x_{1}\right)-f_{\mathrm{em} 2}\left(i_{2}, x_{1}\right)+ \\
& +\left(f_{\mathrm{fr}_{13}}-f_{\mathrm{fr}_{12}}\right) \operatorname{sign} \dot{x}_{3}-F_{N} .
\end{aligned}\right.
$$

Equations of electrical equilibrium (1), (2), and equations of motion of a mechanical system (3) determine the mathematical model of the dynamic state of the vibrational electromechanical system without taking into account the shock interaction, which will be described by a system of differential equations:

$$
\begin{aligned}
& u_{1}(t)=i_{1} r_{1}+\frac{d \psi_{1}\left(i_{1}, x_{1}\right)}{d t} ; \\
& u_{2}(t)=i_{2} r_{1}+\frac{d \psi_{2}\left(i_{2}, x_{1}\right)}{d t} ; \\
& m_{1} \frac{d^{2} x_{1}}{d t^{2}}+k_{1}\left(x_{1}-x_{3}\right)+b_{1}\left(\frac{d x_{1}}{d t}-\frac{d x_{3}}{d t}\right)= \\
& =-f_{\mathrm{em} 1}\left(i_{1}, x_{1}\right)+f_{\mathrm{em} 2}\left(i_{2}, x_{1}\right)-f_{\mathrm{fr}_{13}} \operatorname{sign} \frac{d x_{3}}{d t} \\
& m_{2} \frac{d^{2} x_{2}}{d t^{2}}+k_{2} x_{2}-k_{3}\left(x_{3}-x_{2}\right)+b_{2} \frac{d x_{2}}{d t}- \\
& -b_{3}\left(\frac{d x_{3}}{d t}-\frac{d x_{2}}{d t}\right)=f_{\mathrm{fr}_{23}} \operatorname{sign} \frac{d x_{2}}{d t} ; \\
& -b_{1}\left(\frac{d x_{1}}{d t}-\frac{d x_{3}}{d t}\right)+b_{3}\left(\frac{d x_{3}}{d t}-\frac{d x_{2}}{d t}\right)=f_{\mathrm{em} 1}\left(i_{1}, x_{1}\right)- \\
& m_{3} \frac{d^{2} x_{3}}{d t^{2}}-k_{1}\left(x_{1}-x_{3}\right)+k_{3}\left(x_{3}-x_{2}\right)-\left(f_{\mathrm{fr}_{13}}-f_{\mathrm{fr}}\right) \operatorname{sign} \frac{d x_{3}}{d t}-F_{N} ;
\end{aligned}
$$

Complex mechanical vibrations appear under the action of external periodic forces $f_{\mathrm{em} 1}\left(i_{1}, x_{1}\right)$ and $f_{\mathrm{em} 2}\left(i_{2}, x_{1}\right)$ in a mechanical system.

The real process of motion should be considered as a result of the combined action of complex oscillations of the mechanical system and periodic shock impulses of forces.

The stop and reverse of the striker can be described by an independent system of equalities with respect to the pole system, for example, the return coil:

$$
x_{1}(t)=\left\{\begin{array}{l}
0, \text { at } \delta_{2}^{+}=h_{4}-h_{2} ; \\
h_{4}-h_{2}-\delta_{2}^{+}, \text {at } 0 \leq x_{1} \leq h_{4}-h_{2} ; \\
h_{4}-h_{2}+\left|\delta_{2}^{-}\right|, \text {at } x_{1}>h_{4}-h_{2},
\end{array}\right.
$$

where $\delta_{2}^{+}, \delta_{2}^{-}$- working air gap, formed by the position of the striker relative to the upper pole system of the return coil; $h_{2}$ and $h_{4}$ - fixed coordinates relative to the impact section of the working tool (Figure 1).

At the moment of impact of the striker on the working tool, part of its kinetic energy $T_{1}$ in the form of a shock pulse of forces is transferred to the working tool and further into the deformable medium, while the other part of the kinetic energy $T_{2}$, when the striker rebounds from the working tool, returns to the mechanical system:

$$
\begin{gathered}
T_{1}=\frac{m_{1} v_{1}^{2}}{2}\left(1-k_{\mathrm{reb}}^{2}\right) ; \\
T_{2}=\frac{m_{1} v_{1}^{2}}{2} k_{\mathrm{reb}}^{2}
\end{gathered}
$$

where $k_{\text {reb }}=\frac{\bar{v}_{1}}{v_{1}}$ - striker rebound coefficient; $\bar{v}_{1}$ - velocity of the center of mass of the striker at the end of impact when bouncing off the working tool; $v_{1}$ - velocity of the center of the striker mass at the beginning of the impact.

The component of the spring force of the buffer spring, linearly depending on the position of the striker and the component of the resistance force of the striker, proportional to the speed of its movement, can be described by piecewise linear functions:

$$
\begin{aligned}
& \frac{\partial \Pi}{d x_{1}}=\left\{\begin{array}{l}
0, \text { at } 0 \leq x_{1}<h_{3}-h_{2} ; \\
k_{1}\left(x_{1}-x_{3}\right), \text { at } x_{1} \geq h_{3}-h_{2},
\end{array}\right. \\
& \frac{\partial \Phi}{d \dot{x}_{1}}=\left\{\begin{array}{l}
0, \text { at } 0 \leq x_{1}<h_{3}-h_{2} ; \\
b_{1}\left(\frac{d x_{1}}{d t}-\frac{d x_{3}}{d t}\right), \text { at } x_{1} \geq h_{3}-h_{2},
\end{array}\right.
\end{aligned}
$$


Based on condition (11), let us take into account the fact that the process of energy dissipation under conditions of viscous friction caused by dissipative forces of elastic bonds can arise only when the forces of these bonds work.

In a particular case, the characteristic of the elastic force, being linear on individual sections of the motion, turns out to be generally nonlinear.

In the process of calculation, the authors also assume that the resistance and elastic properties of the deformed medium, provided by the tool movement, depend on the properties of the introduced elastic bonds.

Thus, considering the real process of motion as a result of the combined action of forced, free oscillations and periodic shock impulses of forces, the generalized model of the dynamic state of the electromechanical system (Figure 1) described by equations (4) - (8) under the established conditions of constraints (9) - (11), is reduced to the following system of differential equations:

$$
\begin{aligned}
& u_{1}(t)=i_{1} r_{1}+\frac{d \psi_{1}\left(i_{\mu 1}, x_{1}\right)}{d t} \\
& u_{2}(t)=i_{2} r_{2}+\frac{d \psi_{2}\left(i_{\mu 2}, x_{1}\right)}{d t} ; \\
& i_{1}=i_{\operatorname{los} 1}+i_{\mu 1} ; \quad i_{2}=i_{\operatorname{los} 2}+i_{\mu 2} ; \\
& \int \frac{m_{1} \bar{v}_{1}}{\tau_{\text {и }}}-f_{\text {ет } 1}\left(i_{\mu 1}, x_{1}\right)+f_{\text {em } 2}\left(i_{\mu 2}, x_{1}\right)- \\
& -f_{\mathrm{fr}_{13}} \operatorname{sign} \frac{d x_{1}}{d t} \text {, if } \frac{d x_{1}}{d t}>0, \\
& \text { at } 0 \leq x_{1}<h_{3}-h_{2} \text {; } \\
& m_{1} \frac{d^{2} x_{1}}{d t^{2}}=\left\{\begin{array}{l}
-\tilde{\lambda}_{1}-f_{\mathrm{em} 1}\left(i_{\mu 1}, x_{1}\right)+f_{\mathrm{em} 2}\left(i_{\mu 2}, x_{1}\right)- \\
-f_{\mathrm{fr}_{13}} \operatorname{sign} \frac{d x_{1}}{d t}, \text { at } x_{1} \geq h_{3}-h_{2} ;
\end{array}\right. \\
& -f_{\mathrm{em} 1}\left(i_{\mu 1}, x_{1}\right)+f_{\mathrm{em} 2}\left(i_{\mu 2}, x_{1}\right)- \\
& -f_{\mathrm{fr}_{13}} \operatorname{sign} \frac{d x_{1}}{d t} \text {, if } \frac{d x_{1}}{d t}<0, \\
& \text { at } 0 \leq x_{1}<h_{3}-h_{2} \text {; } \\
& m_{2} \frac{d^{2} x_{2}}{d t^{2}}=-\frac{m_{2} \bar{v}_{2}}{\tau_{\mathrm{p}}}-\tilde{\lambda}_{2}+\tilde{\lambda}_{3}+f_{\mathrm{fr}} \operatorname{sign} \frac{d x_{2}}{d t} \text {; } \\
& m_{3} \frac{d^{2} x_{3}}{d t^{2}}=\left\{\begin{array}{l}
-\tilde{\lambda}_{3}-f_{\mathrm{em} 1}\left(i_{\mu 1}, x_{1}\right)+f_{\mathrm{em} 2}\left(i_{\mu 2}, x_{2}\right)+ \\
+\left(f_{\mathrm{fr}_{13}}-f_{\mathrm{fr}_{23}}\right) \operatorname{sign} \frac{d x_{3}}{d t}-F_{N}, \\
\text { at } 0 \leq x_{1}<h_{3}-h_{2} ; \\
\tilde{\lambda}_{1}-\tilde{\lambda}_{3}-f_{\mathrm{em} 1}\left(i_{\mu 1}, x_{1}\right)+f_{\mathrm{em} 2}\left(i_{\mu 2}, x_{1}\right)- \\
-\left(f_{\mathrm{fr}_{13}}-f_{\mathrm{fr}_{23}}\right) \operatorname{sign} \frac{d x_{3}}{d t}, \text { at } x_{1} \geq h_{3}-h_{2},
\end{array}\right.
\end{aligned}
$$

where $\tilde{\lambda}_{1}=k_{1}\left(x_{1}-x_{3}\right)+b_{1}\left(\frac{d x_{1}}{d t}-\frac{d x_{3}}{d t}\right) \tilde{\lambda}_{2}=k_{2} x_{2}+b_{2} \frac{d x_{2}}{d t}$; $\tilde{\lambda}_{3}=k_{3}\left(x_{3}-x_{2}\right)+b_{3}\left(\frac{d x_{3}}{d t}-\frac{d x_{2}}{d t}\right) ; \quad i_{\operatorname{los} 1}, \quad i_{\operatorname{los} 2}-$ The components of the current loss due to eddy currents and hysteresis in the first and second coils; $i_{\mu 1}, i_{\mu 2}$ - components of the magnetizing current in the first and second coils; $m_{1} \bar{v}_{1}$, $m_{2} \bar{v}_{2}$ - impulses of force at the end of the strike; $\tau_{\mathrm{p}}$ - the time of action of these power pulses.

The obtained system of differential equations of the dynamics of the electromagnetic shock unit allows one to perform a comprehensive analysis of electromechanical processes in transient and quasi-steady operating modes, including the nonlinearity in the magnetic system, the degree of mobility of the inertial masses and the properties of elastic bonds in the mechanical system, accompanied by various kinds of energy losses.

The construction of the structural model of the dynamics of the impact node and the development of the calculation algorithm have been made in accordance with the available recommendations $[3,4]$.

The solution of the field part of the problem was carried out using the standard program of finite element modeling of the magnetic field [3, 4], with the help of an array of values of the reference points of the static flux- $\psi_{\mathrm{em} 1}=f\left(i_{1}, x_{1}\right)$, $\psi_{\mathrm{em} 2}=f\left(i_{2}, x_{1}\right)$ and electromagnetic force $f_{\mathrm{em} 1}=f\left(i_{1}, x_{1}\right)$, $f_{\mathrm{em} 2}=f\left(i_{2}, x_{1}\right)$. Calculation of static parameters $\psi(i, \delta)$ and $f_{\text {em }}(i, \delta)$ was carried out through the integral characteristics of the field in the regime of the block parameters by the weighted average tensor of tension.

The implementation of the mathematical model was carried out by methods of structural modeling in Matlab Simulink.

While developing the calculation algorithm using MatLab Simulink, the function of two arguments of the static parameters of the model was specified using blocks of twodimensional tables.

The simulation results in the form of time diagrams of the process of incorporating the upgraded version of the SC-2 electromagnetic impact node are shown in Figure 3 [2].

The following parameters of the impact knot were used as the initial data of the model: $m_{1}=0,394 \mathrm{kq} ; m_{2}=0,32 \mathrm{kq}$; $m_{3}=3,9 \mathrm{kq} ; \quad k_{1}=120 \cdot 10^{3} \mathrm{~N} / \mathrm{m} ; \quad b_{1}=8 \mathrm{~N} \cdot \mathrm{s} / \mathrm{m}$; $k_{2}=12 \cdot 10^{6} \mathrm{~N} / \mathrm{m} ; \quad b_{2}=0 ; \quad k_{3}=16 \cdot 10^{3} \mathrm{~N} / \mathrm{m}$; $b_{3}=35 \mathrm{~N} \cdot \mathrm{s} / \mathrm{m} ; f_{\text {fr13 }}=3 \mathrm{~N} ; f_{\text {fr } 23}=8 \mathrm{~N} ; F_{\mathrm{N}}=350 \mathrm{~N}$.

Magnetic conductor is made of electrical steel, close in magnetic properties to steel grade 1212. Striker - all-metal is made of structural steel $40 \mathrm{XH}$. The number of turns of the travel coil is $w_{1}=1290$, active resistance is $r_{1}=12,9 \mathrm{Om}$. 
The number of turns of the return coil is $w_{2}=1540$, active resistance is $r_{2}=23,2 \mathrm{Om}$.

The effective value of the voltage of a single-phase source is $U=220 \mathrm{~B}$.

To assess the adequacy of the model created, a comparison is made among the calculated values of the impact energy parameters, the current value of the current, the power consumption; net power, efficiency and power factor with the indices obtained on the physical model (Table 1).

The relatively small discrepancy between the quantitative results of the calculations and the experimental data, which amounts to $4 \ldots 6 \%$, allows us to conclude that the model is adequate.

It should be noted that the relatively good agreement between the calculated and experimental parameters largely depends on the accuracy of the analogs of the mechanical characteristics of the model, in particular the damping properties of elastic bonds and the forces of dry sliding friction, which most developers neglect to simplify the model.

\section{CONCLUSION}

The article provided a mathematical model of the dynamic state of a two-coil synchronous impact machine with a springloaded reverser of the striker, which offers wide opportunities for a comprehensive analysis of interrelated electromechanical processes during the excitation of periodic shock impulses of forces and interaction with a deformed medium.

The peculiarity of the model is the ability to take into account a large number of interrelated factors due to the speed of the striker's movement and the frequency of impact forces pulses, the degree of mobility of inertial masses and the properties of elastic bonds of the mechanical system, as well as the nonlinearity of the characteristics of magnetic materials, accompanied by various kinds of energy losses.

The verification of the mathematical model is performed by comparing the calculated values of the output indicators with the results obtained on the physical model. The difference between the calculated and experimental values does not exceed $4 \ldots 6 \%$, which is customary for engineering practice.

TABLE 1. Comparison of simulation results

\begin{tabular}{|l|c|c|}
\hline Indicator values & Calculation & Experiment \\
\hline Impact energy, Joule & 5,6 & 5,8 \\
\hline $\begin{array}{l}\text { The effective value of the } \\
\text { current, Ampere }\end{array}$ & 4,42 & 4,68 \\
\hline Power consumption, Watt & 737 & 783 \\
\hline Effective power, Watt & 280 & 290 \\
\hline Efficiency, \% & 0,38 & 0,37 \\
\hline Power factor, $\cos \varphi$ & 0,44 & 0,42 \\
\hline
\end{tabular}

\section{References}

[1] V.P. Pevchev, "The superexitation and efficiency relation in a shortstroke pulsed electromagnetic motor of a seismic source", Journal of Mining Science, 2010, vol. 46, pp. 656-665, June 2010.

[2] S.N. Kuchankov, E.M. Timoshenko, "Nonsteady thermal conditions of electromagnetic motors in shock-type equipment", Journal of Mining Science, 1998, vol. 34 . pp. 148-152, February 1998.

[3] V.Y. Neiman, L.A. Neiman, A. S. Shabanov, "A simplified Calculation of the Intermittent periodic operating regime of an electromagnetic", Russian Electrical Engineering, , vol. 85, pp. 757-760, December 2014.

[4] L.A. Neiman, V.Y. Neiman, Linear synchronous electromagnetic machines for lowfrequency impact technologies", Russian Electrical Engineering, vol. 85, pp. 752-756, December 2014.

[5] V.P. Pevchev, "Principal dimensions of the short-stroke electromagnetic motor for a seismic wave generator", Journal of Mining Science, 2010, vol. 45, pp. 372-381, April 2010. 